\title{
Pulmonary artery pseudoaneurysm in a patient with Behçet disease
}

\author{
Yong Han Yoon, MD, ${ }^{a}$ Kwang Ho Kim, MD, ${ }^{a}$ Wan Ki Baek, MD, Jung Taek Kim, MD, ${ }^{a}$ Kuk Hee Shon, MD, \\ Young Sam Kim, MD, ${ }^{a}$ Hae Seung Han, MD, and Jae Hwa Cho, MD, ${ }^{c}$ Incheon, Korea
}

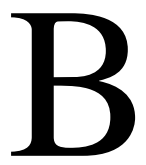

ehçet disease is a rare and chronic disorder of unknown cause characterized by the inflammation of blood vessels; pulmonary involvement occurs in only about $1 \%$ to $7 \%$ of patients. ${ }^{1-4}$ Of the pulmonary manifestations, the pulmonary arteries are second to the aorta as the most common site of arterial involvement. Pulmonary artery aneurysm, in particular, has a poor prognosis and is one of the leading causes of death in patients with Behçet disease. Mean survival after the onset of hemoptysis was reported to be about 10 months in one study of patients with Behçet disease and pulmonary artery aneurysm. ${ }^{5} \mathrm{We}$ report a case involving a young female patient who presented with a variety of symptoms, leading to a diagnosis of Behçet disease and treatment for pulmonary artery pseudoaneurysm after appropriate investigations and surgical intervention. After a right lower lobe lobectomy, no further hemoptysis and skin lesions have occurred 20 months postoperatively.

\section{Clinical Summary}

A 35-year-old woman was admitted to Inha University Hospital, Incheon, Korea, because of high fever and cough for the previous 2 months. For the previous 3 years, she had recurrent episodes of oral ulceration. A chest radiograph taken 2 months before admission showed a mass-like lesion in the right lower lung field. Physical examination revealed several small, ulcerated lesions on her external genitalia. However, her breath sounds were clean, and heart sounds were regular and without murmur. Pathergy test results were positive, but the results of other blood tests were within normal limits. The chest radiograph showed a round mass $6 \times 7 \mathrm{~cm}$ in diameter in the right lower lung field (Figure 1). The computed tomographic scan of the chest, enhanced through intravenous injection of contrast media, showed that the mass originated from the right lower pulmonary artery. Behçet disease with pulmonary artery aneurysm was investigated on the basis of the aforementioned findings.

Seven days after admission, the patient had a high fever with sudden hemoptysis of about $30 \mathrm{~mL}$. The follow-up chest radiograph showed the lung mass had increased in size since admission.

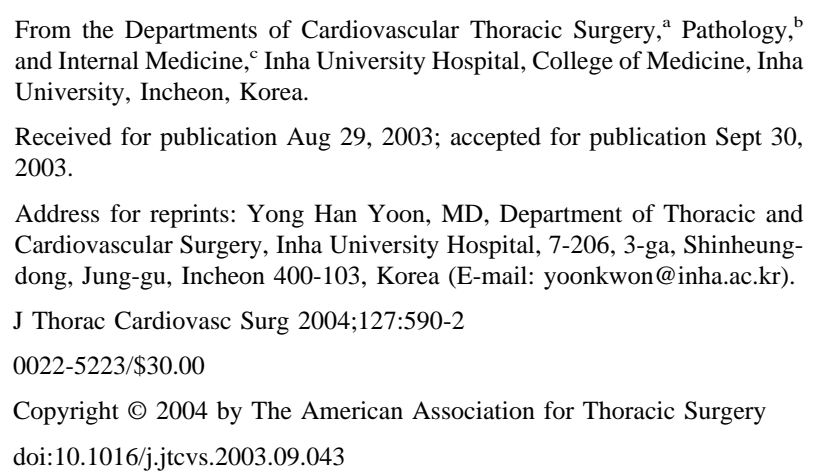

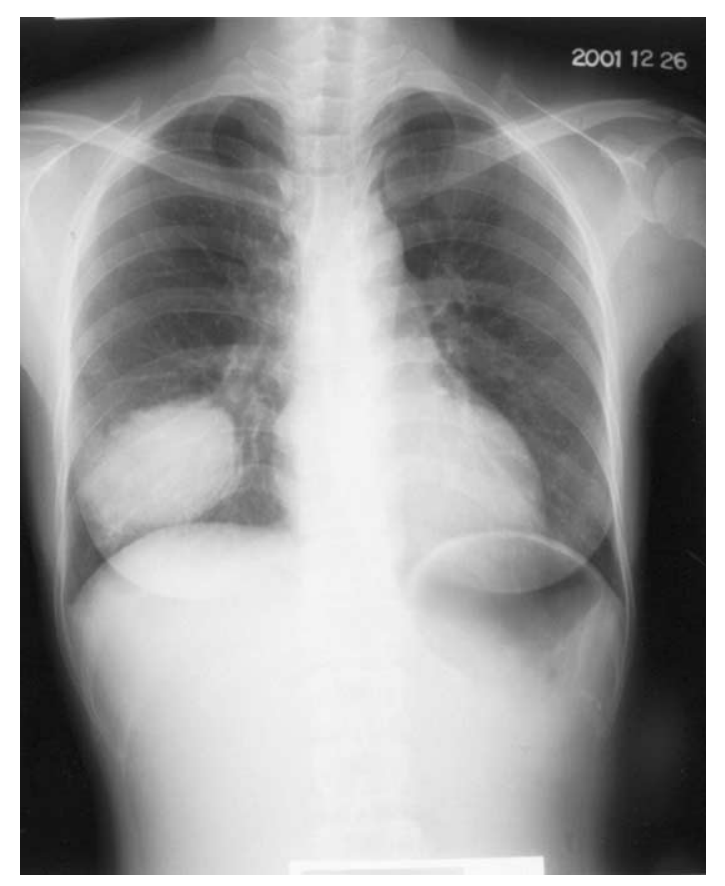

Figure 1. The posteroanterior chest radiograph showed a round mass $6 \times 7 \mathrm{~cm}$ in diameter in the right lower lung fields.

A magnetic resonance imaging study confirmed a ruptured aneurysm of the right lower lobar pulmonary artery, indicating a pulmonary artery pseudoaneurysm (Figure 2). A lobectomy of the right lower lobe was performed, and a cystic mass containing blood and measuring $4 \times 4 \times 4 \mathrm{~cm}$ in size was found in the superior segment of the lobe. The patient's postoperative course was uneventful. The pathologic findings revealed that the cystic lesion was a ruptured aneurysm of the large pulmonary artery. Elastic staining of this involved pulmonary artery showed that a portion of the wall was destroyed and replaced by inflamed fibrous tissue. The cellular infiltrate of the vessel wall was found to be predominantly composed of lymphocytes, macrophages, neutrophils, and eosinophils. Periadventitial fibrosis was predominant, and an organized thrombus with recanalization of a branch of the pulmonary artery was also found. Vasculitis in this case involved the large pulmonary artery only because vessels of different types and sizes were not affected. A pulmonary artery bronchial fistula formed because of the spread of inflammation and necrosis from the fibrous wall of an elastic pulmonary artery aneurysm into an adjacent bronchus (Figure 3). Seven days postoperatively, the chest tube was removed. During the postoperative course, skin lesions developed as a result of trauma, such as venipuncture and arterial puncture. The patient was administered a course of corti- 


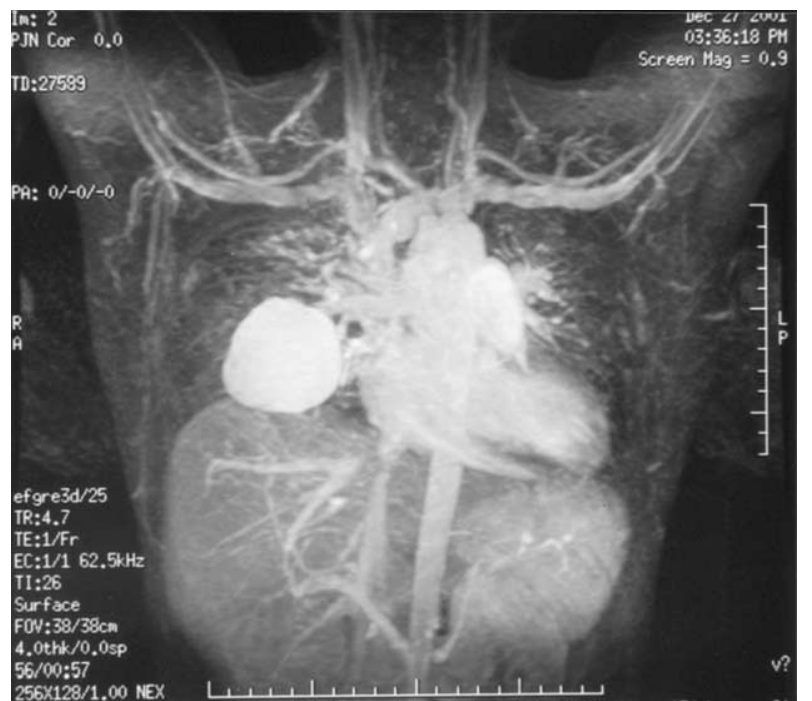

Figure 2. Chest magnetic resonance imaging showing the ruptured aneurysm with the lesion that was interpreted as representing pulmonary artery pseudoaneurysm.

costeroids, and no further hemoptysis and skin lesions occurred in the 20 months after the lobectomy.

\section{Discussion}

The vascular system is affected in $25 \%$ of patients with Behçet disease, with specifically pulmonary manifestations occurring in between only $1 \%$ and $7 \%$ of patients. ${ }^{1}$ Pulmonary artery aneurysms, in particular, have a poor prognosis and are one of the leading causes of patient death in Behçet disease. Unfortunately, no prospective studies have examined the true prevalence of the pulmonary manifestations of the disease. Aneurysms of pulmonary arteries are very rare. Aortic manifestations are the most common vascular involvement, and pseudoaneurysms in this area have been reported. ${ }^{6,7}$ Pulmonary artery pseudoaneurysms have not been reported in the literature until our case study. Generally, pulmonary artery pseudoaneurysms occur as a result of erosion of the artery by mycotic aneurysms derived from right-side bacterial endocarditis or infected thrombi from peripheral veins; trauma and chronic pulmonary hypertension, particularly that associated with congenital heart disease, in which a left-to-right shunt develops; and septic emboli. Behçet disease should be included in the listing of diseases causing aneurysms of the large pulmonary arteries. Important complications of pulmonary angitis in Behçet disease include vascular thromboses, pulmonary infarction, bronchial erosion by pulmonary arteries, and pulmonary artery aneurysm. ${ }^{1,2,8}$ Pulmonary vasculitis in Behçet disease is a unique clinical and pathologic picture. ${ }^{1-4,8}$ Pulmonary artery aneurysms are located mainly in the right lower lobar arteries, followed by the right and left main pulmonary arteries. ${ }^{8}$ The pulmonary manifestations appear, on average, 3.6 years after the first extrapulmonary manifestation. Hemoptysis is the major pulmonary manifestation and is reported to occur in $77 \%$ of reported cases. Hemoptysis might result from the acute inflammatory destruction of elastic and muscular pulmonary arteries, the rupture of pulmonary artery

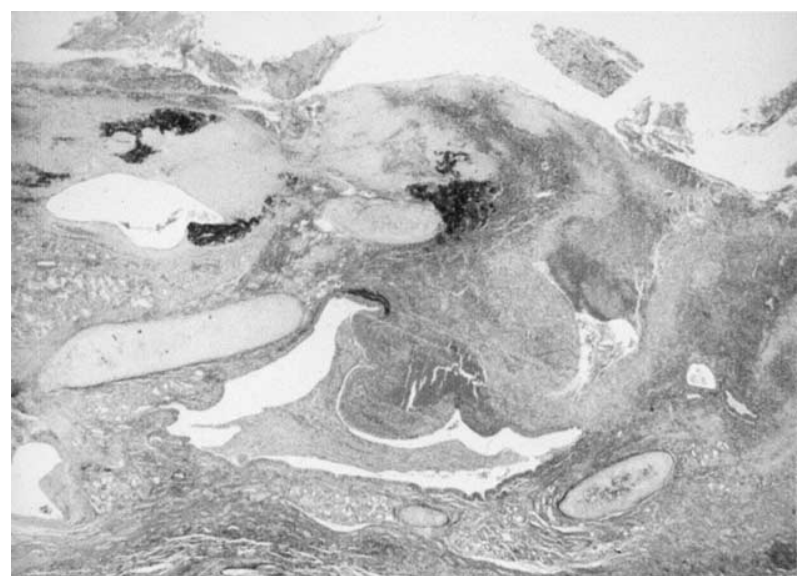

Figure 3. Spread of inflammation and necrosis from the fibrous wall of an elastic pulmonary artery aneurysm into an adjacent bronchus leads to the formation of a pulmonary artery bronchial fistula. (Elastic staining, original magnification $12.5 \times$.)

aneurysms, pulmonary infarcts, erosion of pulmonary artery aneurysm into adjacent bronchi, and finally, the extensive inflammatory disruption of alveolar capillaries. ${ }^{1-5,8}$ In our case hemoptysis resulted from the erosion of the pulmonary artery pseudoaneurysm into adjacent bronchi. One characteristic of Behçet disease is that vasculitis can occur in different types of pulmonary vessels and might involve large and small vessels simultaneously or separately in the course of the disease.

Chest x-ray films showed abnormal findings in $90 \%$ of the cases. Helical computed tomography is currently the method of choice for confirming the diagnosis because it provides an excellent vascular image, requiring only a small quantity of contrast material. Magnetic resonance imaging is also helpful in the diagnosis of pulmonary artery aneurysms and pseudoaneurysms.

Currently, the mainstay of treatment is immunosuppressants, especially when introduced in the early stages before irreversible damage to the arterial wall develops. Anticoagulant therapy holds potential hazards to patients with aneurysmal dilatation of the pulmonary blood vessels. When the disease is localized to one area of the lung, surgical excision, followed by medical treatment, is indicated to prevent fatal pulmonary hemorrhage.

In patients with massive hemoptysis, urgent surgical resection might be necessary and after postoperative steroid treatment because perioperative steroid therapy has been suggested to reduce the risk of complications.

In our patient no further hemoptysis occurred 20 months after the lobectomy.

\section{References}

1. Slavan RE, de Groot WJ. Pathology of the lung in Behçet's disease case report and review of the literature. Am J Surg Pathol. 1981;5: $779-88$.

2. Erkan F, Gul A, Tasali E. Pulmonary manifestation of Behçet's disease. Thorax. 2001;56:572-8. 
3. Raz I, Okon E, Cheajek-Shaul E. Pulmonary manifestation of Behçet's disease. Chest. 1989;95:585-9.

4. Bang DS, Lee JH, Lee ES, et al. Epidermiologic and clinical survey of Behçet's disease in Korea: the first multicenter study. J Korean Med Sci. 2001;16:L615-8.

5. Erkan F. Pulmonary involvement in Behçet's disease. Curr Opin Pulm Med. 1999;5:314-8.

6. Okita Y, Ando M, Minatoya K, et al. Pseudoaneurysm of aortic arch, right subclavian artery and abdominal aorta in a patient with Behçet's disease. J Vasc Surg. 1998;28:723-6.

7. Nonaka K, Makuuchi H, Naruse J, et al. Pseudoaneurysm of aortic arch and rupture into pericardium, a case report of successful surgical management. Jpn J Thorac Cardiovasc Surg. 1998;46:772-6.

8. Tunaci A, Berkmen YM, Gokmen E. Thoracic involvement in Behçet's disease: pathologic, clinical, and imaging features. Am J Roentgenol. 1995;164:51-6.

\title{
Methylene blue for lithium-induced refractory hypotension in off-pump coronary artery bypass graft: Report of two cases
}

\author{
Donatella Sparicio, MD, Giovanni Landoni, MD, Federico Pappalardo, MD, Martina Crivellari, MD, Elisa Cerchierini, MD,
} Giovanni Marino, MD, and Alberto Zangrillo, MD, Milano, Italy

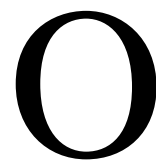
ff-pump surgery is gaining popularity for coronary artery bypass grafting (CABG). We report the perioperative management of 2 patients who self-assumed lithium and had refractory hypotension during beating heart surgery. Both patients had a dramatic hemodynamic improvement after receiving methylene blue.

\section{Clinical Summary}

Two men (57 and 68 years, each weighing $72 \mathrm{~kg}$, height 180 and $186 \mathrm{~cm}$ ) with coronary artery disease and ejection fraction greater than $50 \%$ were scheduled for elective off-pump CABG. Medical history included depression on treatment with benzodiazepines and lithium carbonate (300 mg every 8 hours). They were both on treatment with beta-blockers (atenolol and carvedilol), calcium antagonist (amlodipine), nitrates, and aspirin; patient A also used an angiotensin-converting enzyme inhibitor. Both patients had an unnoticed self-administration of lithium the morning before surgery.

Standard premedication and anesthesia were administered. Target coronary vessels were mechanically stabilized and posterior vessel presentation obtained with a suspension stitch placed in the middle of the 4 pulmonary veins. Both patients received an in situ internal thoracic artery graft to the left anterior descending artery and a saphenous vein graft to the posterior descending and obtuse marginal arteries. Hypotension ensued after pericardial opening and anastomosis. Arterial blood pressure, 80/50 mm Hg (mean

From the Department of Cardiovascular Anesthesia, Vita-Salute University of Milan, IRCCS San Raffaele Hospital, Milan, Italy.

Received for publication June 16, 2003; accepted for publication Sept 12, 2003.

Address for reprints: Donatella Sparicio, MD, Via Olgettina 60, 20132 Milano, Italy (E-mail: Sparicio.donatella@hsr.it).

J Thorac Cardiovasc Surg 2004;127:592-3

$0022-5223 / \$ 30.00$

Copyright @ 2004 by The American Association for Thoracic Surgery

doi:10.1016/j.jtcvs.2003.09.019 arterial pressure $=60 \mathrm{~mm} \mathrm{Hg}$ ), was not responsive to etilephrine boluses, atrial pacing, dopamine infusion $(10 \mu \mathrm{g} / \mathrm{kg} / \mathrm{min})$, steep Trendelenburg position, fluid loading, and opening of both pleura; nevertheless, patients' stability, in the absence of arrhythmias or ST-T changes, maintenance of diuresis, and an optimal cooperation between anesthesiologist and surgeon, permitted concluding the procedure without converting to cardiopulmonary bypass. Intraoperative transesophageal echocardiography demonstrated normal biventricular function without new segmental acinesia. In the hypothesis of vasoplegia both patients received methylene blue $(1.5 \mathrm{mg} / \mathrm{kg}$ intravenously) in 20 minutes with a rapid improvement of mean arterial pressure $(80 \mathrm{~mm} \mathrm{Hg})$.

At the end of the operation, the patients were transferred to the intensive care unit and had an uneventful postoperative course.

\section{Discussion}

Lithium $\left(\mathrm{Li}^{+}\right)$, the primary drug used for the treatment of bipolar (maniac-depressive) disorders, may induce prolonged sleep time after barbiturate anesthesia, enhanced neuromuscular blockade after muscle relaxant administration, and cardiac conduction abnormalities. ${ }^{1}$ The mechanism of action of $\mathrm{Li}^{+}$as a mood-stabilizing agent remains unknown. Therapeutic doses of lithium interfere with the metabolism of cathecolamines that are involved in the pathophysiology of the troubles of humor; $\mathrm{Li}^{+}$also inhibits the liberation of norepinephrine and dopamine from the nervous terminations, strengthens the liberation of serotonin, and increases the presynaptic reuptake and storage of catecholamines. Furthermore, it has interactions with drugs like angiotensin-converting enzyme inhibitors (which reduce $\mathrm{Li}^{+}$clearance with possible $\mathrm{Li}^{+}$ toxicity) and beta-blockers (which enhance the central depression induced by lithium).

In light of these pharmacodynamic properties, in these patients we suspected that chronic lithium therapy played a role in the unresponsiveness to vasoconstrictor agents. Lithium should be discontinued before surgery, at least the day of the operation, especially when high blood pressures are requested during surgery. $^{2}$

Methylene blue inhibits the enzyme guanylate cyclase, avoiding the cyclic guanosine 3'5'-monophosphate-dependent vasore- 